\title{
Detailed analysis of the induction period of polymer crystallization by depolarized light scattering
}

$\operatorname{AUTHOR}(\mathrm{S}):$

Matsuba, G; Kaji, K; Kanaya, T; Nishida, K

\section{CITATION:}

Matsuba, G ... [et al]. Detailed analysis of the induction period of polymer crystallization by depolarized light scattering. Physical Review E 2002, 65(6): 061801.

ISSUE DATE:

2002-06

URL:

http://hdl.handle.net/2433/39904

RIGHT:

Copyright 2002 American Physical Society 
PHYSICAL REVIEW E, VOLUME 65, 061801

\title{
Detailed analysis of the induction period of polymer crystallization by depolarized light scattering
}

\author{
Go Matsuba, ${ }^{*}$ Keisuke Kaji ${ }^{\dagger}$ Toshiji Kanaya, and Koji Nishida \\ Institute for Chemical Research, Kyoto University, Gokasho, Uji, Kyoto-fu 611-0011 Japan
}

(Received 27 December 2001; published 10 June 2002)

\begin{abstract}
In order to clarify the structure formation processes in the induction period of polymer crystallization the annealing time dependence of depolarized light-scattering (DPLS) intensities has been investigated as a function of crystallization temperature for poly(ethylene terephthalate), poly(ethylene naphthalate), syndiotactic polystyrene, and isotactic polystyrene. It is found that the induction period may be separated into three stages: the first stage where the DPLS intensity hardly changes with time, the second stage where the intensity increases exponentially, and the third stage where it levels off. Considering that the DPLS provides information about the degree of parallel orientation of rigid polymer segments, the first stage whose time length depends on the annealing temperature may be assigned to a process where the polymer chains begin to partially assume a rigid conformation, generally a helical structure being almost the same as the structure in the corresponding crystal. This process is limited to a time when the average length of the rigid segments attains a critical value given by a Shimada, Doi, and Okano theory [J. Chem. Phys. 88, 7181 (1988)] above which spinodal decomposition (SD) is caused. The second and third stages correspond to the early and late stages of SD, respectively, which was confirmed by small-angle x-ray scattering measurements. The apparent activation energies obtained from the temperature dependence of the DPLS intensities for the three stages were 35-40, 25-50, and $180-400 \mathrm{~kJ} / \mathrm{mol}$, respectively, for all the polymers. The large apparent activation energies for the late stage of SD is discussed within a framework of Binder and Stauffer's theory [Phys. Rev. Lett. 33, 1006 (1974)].
\end{abstract}

DOI: 10.1103/PhysRevE.65.061801

PACS number(s): 61.41.+e, 68.65.-k, 61.20.Lc

\section{INTRODUCTION}

Polymer crystallization processes have been studied for a long time. Of these, what happens during the induction period before crystal nucleation is one of the important unsolved problems in polymer physics. More than ten years ago, we discovered a surprising phenomenon using smallangle x-ray scattering (SAXS) [1-6], small-angle neutron scattering $[3,5,6]$, and depolarized light-scattering (DPLS) [3-6] techniques that a spinodal decomposition (SD) type of microphase separation, whose characteristic wavelength is longer than the so-called long period, actually occurs during the induction period of crystallization just above the glass transition temperature $T_{g}$ for poly(ethylene terephthalate) (PET). Such spinodal decomposition has been understood based on a kinetic theory for the isotropic-to-nematic transition of polymer liquid crystal by Shimada, Doi, and Okano [7-10]. They showed that the SD-type microphase separation is caused by orientation fluctuations of rigid polymer segments which are coupled with density fluctuations. In this connection, we also investigated the crystallization processes just above the glass transition temperature $T_{g}$ for syndiotactic polystyrene (sPS) [11], isotactic polystyrene (iPS) [12], and poly(ethylene naphthalate) (PEN) [13,14], where we have successfully showed by using time-resolved Fourier transform infrared (FTIR) spectroscopic and depolarized

\footnotetext{
*Present address: Polymers Division, National Institute of Standards \& Technology, 100 Bureau Drive, Gaithersburg, MD 20899-8543.

${ }^{\dagger}$ Author to whom correspondence should be addressed. Present address: Institute for Chemical Research, Kyoto University, Uji, Kyoto-fu, 611-0011, Japan. FAX: +81-(0)774-38-3146.
}

light-scattering (DPLS) techniques that the SD is triggered by the extension of rigid segments caused by the conformational change from an amorphous to crystalline one. As was described above, the structure formation in the early stage of SD could be explained using the kinetic theory by Shimada, Doi, and Okano [7-10] for the isotropic-to-nematic transition of liquid-crystalline polymer. However, the time evolution of density fluctuations in the late stage of SD due to orientation fluctuations has never been explained.

The aim of this paper is to experimentally clarify the structure formation in the induction period of crystallization, especially the orientational ordering process of polymer chains, when the polymer is crystallized just above $T_{g}$ from the glassy state. For this purpose, we have performed DPLS measurements on PEN, PET, sPS, and iPS when they were annealed just above $T_{g}$ from the glassy state. We calculated the apparent activation energy with Arrhenius plot from the annealing temperature and time dependence of the integrated intensity of time-resolved DPLS.

\section{EXPERIMENT}

The polymers used for this paper were isotactic polystyrene (iPS), syndiotactic polystyrene (sPS), poly(ethylene terephthalate) (PET), and poly(ethylene naphthalate) (PEN). The number-average molecular weights $M_{n}$ of these samples are $4.0 \times 10^{5}, 2.9 \times 10^{5}, 4.5 \times 10^{4}$, and $6.7 \times 10^{4}$, respectively, and the polydispersity, $M_{w} / M_{n}$, of every sample is about two. The glass transition temperatures were determined with Perkin-Elmer DSC-7 at a scanning rate $5{ }^{\circ} \mathrm{C} / \mathrm{min}$ and obtained as $100^{\circ} \mathrm{C}$ for iPS and sPS, $75^{\circ} \mathrm{C}$ for PET, and $110^{\circ} \mathrm{C}$ for PEN. Amorphous thin films of these polymers were made by quenching their melts into ice water after being kept for $5 \mathrm{~min}$ at about $40^{\circ} \mathrm{C}$ above $T_{m}$ to remove the 


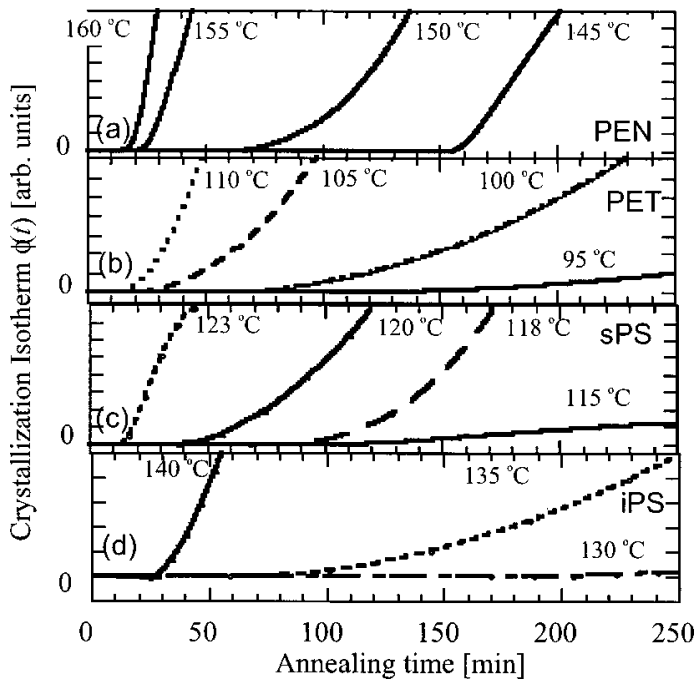

FIG. 1. Annealing time dependence of crystallization isotherm $\phi(t)$ for PEN (a), PET (b), sPS (c), and iPS (d) when jumped to given annealing temperatures from the glassy state.

memory in the polymer samples. In order to remove water, all the samples were dried for one day under vacuum at room temperature.

The time-resolved DPLS measurements were also carried out under annealing conditions just above $T_{g}$ for all the samples. The samples were irradiated by a plane-polarized He-Ne laser $(\lambda=632.8 \mathrm{~nm})$ on a hot stage and the scatteredlight intensity under depolarized conditions was recorded by a photodiode array system at an interval of $0.5 \mathrm{~min}$.

\section{RESULTS}

\section{Estimation of the induction period of crystallization}

Figure 1 shows the annealing time $t$ dependence of crystallization isotherm $\phi(t)$ for PEN (a), PET (b), sPS (c), and iPS (d) measured by differential scanning calorimetry (DSC) as a function of annealing temperature above $T_{g}$. The crystallization isotherm was calculated according to the following equation:

TABLE I. Lengths $t_{\text {ind }}$ of the induction period determined by DSC for PET, PEN, sPS, and iPS as a function of annealing temperature.

\begin{tabular}{crrcrr}
\hline \hline Polymer & $T\left({ }^{\circ} \mathrm{C}\right)$ & $\begin{array}{c}t_{\text {ind }} \\
(\mathrm{min})\end{array}$ & Polymer & $T\left({ }^{\circ} \mathrm{C}\right)$ & $\begin{array}{c}t_{\text {ind }} \\
(\mathrm{min})\end{array}$ \\
\hline \multirow{2}{*}{ PET } & 95 & 150 & PEN & 145 & 150 \\
& 100 & 60 & & 150 & 70 \\
& 105 & 20 & & 155 & 25 \\
& 110 & 10 & & 160 & 10 \\
\multirow{5}{*}{ SPS } & 115 & 110 & iPS & 130 & 150 \\
& 118 & 65 & & 135 & 70 \\
& 120 & 30 & & 140 & 25 \\
& 123 & 10 & & & \\
\hline \hline
\end{tabular}

$$
\phi(t)=\frac{\int_{0}^{t}\left(d H_{t} / d t\right) d t}{\int_{0}^{\infty}\left(d H_{t} / d t\right) d t},
$$

where $d H_{t} / d t$ is the rate of evolution of heat. During the induction period, neither exotherm nor endotherm is observed at all the conditions. For example, in the case of PEN [Fig. 1(a)] the so-called induction period of crystallization is $10,25,70$, and 150 min when annealed at 160, 155, 150, and $145^{\circ} \mathrm{C}$, respectively. Table I summarizes the induction period $t_{\text {ind }}$ as a function of annealing temperature for all the polymers.

\section{Parallel orientational ordering from DPLS measurements}

According to Koberstain, Russel, and Stein [15] the Rayleigh factor $R_{+}(q)$ for depolarized light scattering from solids having randomly correlated orientation fluctuations can be expressed by

$$
R_{+}(q)=\left(\frac{\omega}{c}\right)^{4} \frac{\left\langle\delta^{2}\right\rangle}{15} \int_{0}^{\infty} g(r) \frac{\sin (q r)}{q r}(4 \pi r) d r,
$$

where $q(=4 \pi n \sin \theta / \lambda)$ is the length of scattering vector, $n$, $2 \theta$ and $\lambda$ being the refractive index of the medium, the scattering angle, and the wavelength of light, respectively, $\omega$ is the angular frequency of incident radiation, $c$ is the velocity of light, $\left\langle\delta^{2}\right\rangle$ is the mean-square anisotropy, and $g(r)$ is the function of orientation defined as $g(r)=\left(3\left\langle\cos ^{2} \phi_{i j}\right\rangle_{r}-1\right) / 2$, where $\phi_{i j}$ is the angle between the optical axes of the $i$ th and $j$ th elements [15]. The integrated intensity due to the orientation fluctuations $I_{+}$is given by

$$
I_{+}=\int_{0}^{\infty} R_{+}(q) q^{2} d q=\frac{2 \pi^{2}}{15}\left(\frac{\omega}{c}\right)^{4}\left\langle\delta^{2}\right\rangle .
$$

These Eqs. (2) and (3) give us a basis for interpretations of the results on the depolarized light-scattering measurements.

Figure 2 shows the semilogarithmic expression of time evolution of the DPLS intensity of PEN when annealed at $150{ }^{\circ} \mathrm{C}$ as a function of scattering vector $q$. The induction period at this condition is about $70 \mathrm{~min}$ as seen from Fig. 1(a). The intensity of DPLS increases rapidly even in the induction period of crystallization, suggesting that the parallel ordering of polymer segments proceeds before crystal nucleation. We also notice that such ordered domains are much smaller than the wavelength of He-Ne laser light $(\lambda$ $=632.8 \mathrm{~nm}$ ) because the DPLS profiles during the induction period is almost independent of $q$. After the crystal nucleation, the scattering profiles become $q$ dependent, indicating that the size of orientated domains or their aggregates become comparable to the $q$ range.

Figure 3 shows the time dependence of the integrated intensity of DPLS for PEN films when annealed at $150{ }^{\circ} \mathrm{C}$. In the first stage, or the very early stage until about $2.0 \mathrm{~min}$, the growth rate of the integrated intensity is very slow, and the intensity of the IR band originated from noncrystalline trans conformation hardly increases as described in the previous 


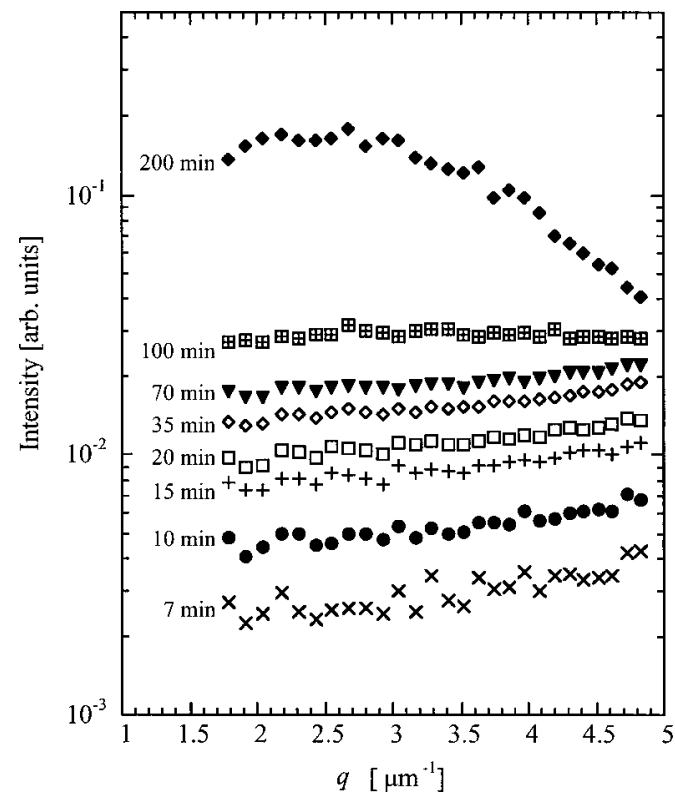

FIG. 2. Depolarized light-scattering profiles $I(q)$ of PEN film when annealed at $150{ }^{\circ} \mathrm{C}$ as a function of annealing time.

paper [14]. In the second stage between 2 and $10 \mathrm{~min}$, the integrated intensity increases exponentially. This is one of characteristic features of SD in the kinetic theory of isotropic-to-nematic transition of liquid crystals [7-10] as well as of the early stage (SD) in the usual phase separation theory by Cahn and Hilliard $[16,17]$. In the third stage between 10 and $70 \mathrm{~min}$, the integrated intensity curve tends to level off. The cause for the leveling off may be considered due to the entanglement effect which suppresses the orientation of rigid segments. After the initiation of crystallization at $70 \mathrm{~min}$, which was determined by DSC measurements

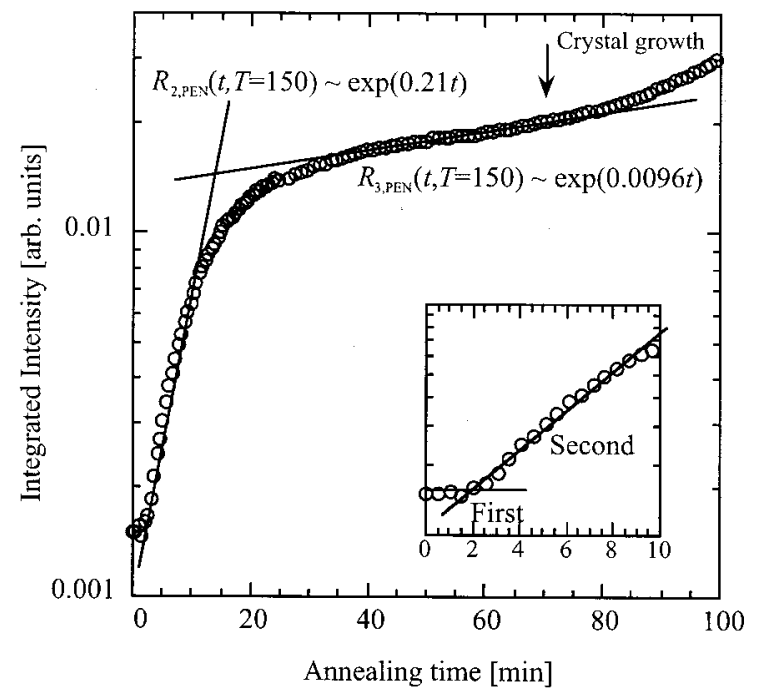

FIG. 3. Annealing time dependence of the integrated intensity of depolarized light scattering (DPLS) for PEN when annealed at $150{ }^{\circ} \mathrm{C}$. The inset is the enlargement of the first and second stages of the induction period.

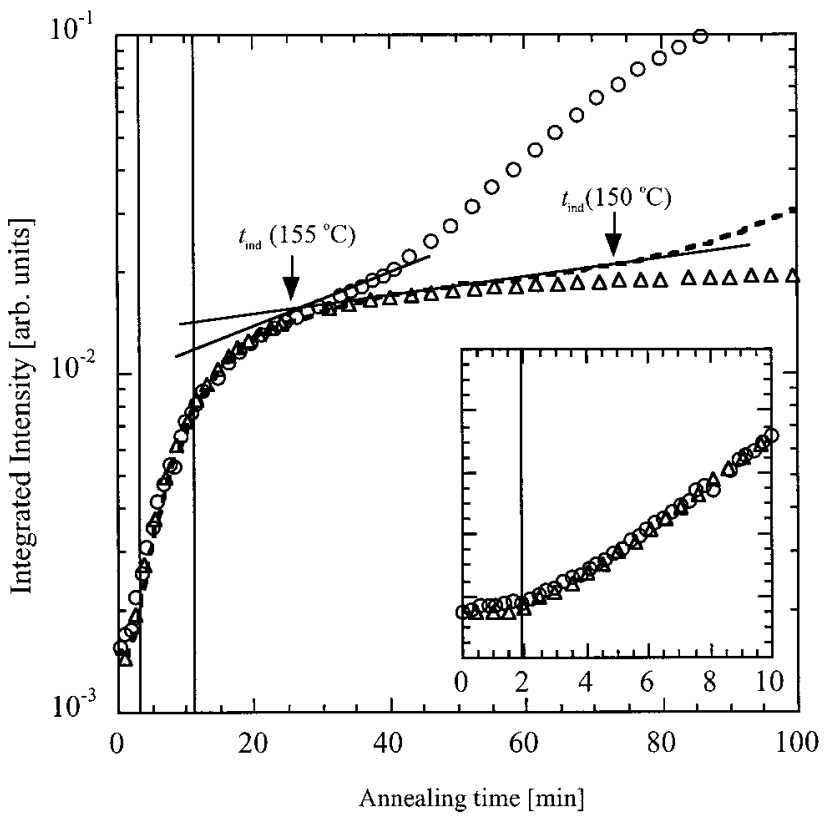

FIG. 4. Annealing time dependence of the integrated intensity of DPLS for PEN as a function of annealing temperature: $155(\bigcirc)$, $150(\cdots)$, and $145^{\circ} \mathrm{C}(\triangle)$. Arrows indicate the times at which crystallization begins though that for $145^{\circ} \mathrm{C}$ is out of the scale. The inset is the enlargement until the initial $10 \mathrm{~min}$.

(Table I), the integrated intensity also begins to increase again; this may be due to the growth of oriented crystalline domains. From the time dependence of the integrated DPLS intensity we can conclude that the induction period of crystallization is separated into three stages.

Figure 4 shows the annealing time dependence of the integrated intensity of DPLS for PEN films as a function of annealing temperature. Here, the data for $150{ }^{\circ} \mathrm{C}$ in Fig. 3 is also shown with a dotted line. The arrows indicate the starting times of crystallization determined by DSC for 150 and $155^{\circ} \mathrm{C}$ though the time $(150 \mathrm{~min})$ for $145^{\circ} \mathrm{C}$ is not shown here since it is out of the scale. The two vertical lines reveal that the induction period can be separated into three stages. The time evolution of the integrated intensity until $25 \mathrm{~min}$ before crystallization is almost independent of annealing temperature, but after $25 \mathrm{~min}$ the integrated intensity is clearly annealing temperature dependent. It increases more rapidly at higher temperatures, which may be due to the increase in orientational diffusion rate of larger rigid segments. On the other hand, we have studied the time evolution of density fluctuations of a PEN glass when it was annealed at $155^{\circ} \mathrm{C}$ by SAXS [13], revealing that the structure formation process during the induction period can be explained by a mechanism of spinodal-decomposition (SD)-type microphase separation. Taking account of this result, it is presumed that the second stage and the third stage correspond to the early stage and the late stage of SD, respectively. Actually, the time evolution of the integrated intensity in the second stage shows an exponential growth which is characteristic of the early stage of SD. However, we cannot confirm the third stage because we do not know how the orientation fluctuations grow with time in the late stage. 


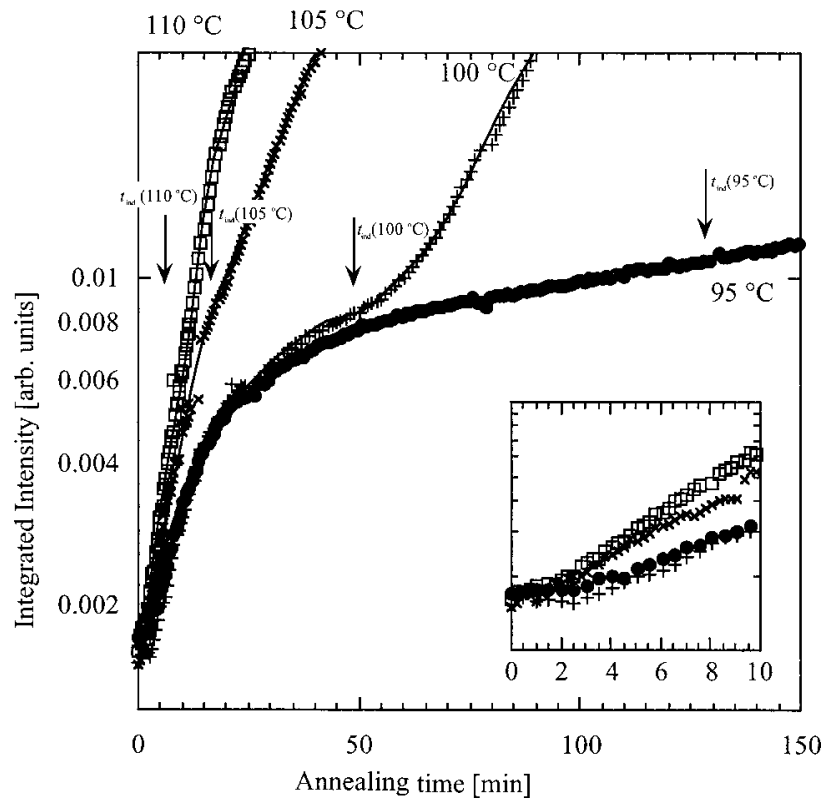

FIG. 5. Annealing time dependence of the integrated intensity of DPLS for PET as a function of annealing temperature: $110(\bigcirc), 105$ $(\times), 100(+)$, and $95^{\circ} \mathrm{C}(\mathbf{)})$. Arrows indicate the times at which crystallization begins. The inset is the enlargement until the initial $10 \mathrm{~min}$.

Similar experiments as a function of annealing temperature have been carried out for PET, sPS, and iPS as well. In every case, the DPLS intensity was independent of the scattering vector $q$ and increases with annealing time in the induction period, suggesting that the sizes of orientated domains are much smaller than the wavelength of the used light and that the parallel orientation of polymer chain segments proceeds before crystal nucleation, respectively. Hence, as an orientation index of chain segments, we can use the integrated intensity in the present $q$-range $I_{+}$[Eq. (3)] also for these polymers. Figures 5, 6, and 7 show the time and temperature dependence of the integrated intensity of DPLS intensity for PET [4,6], sPS [7], and iPS [8] when they were annealed just above $T_{g}$. The arrows in these figures indicate the starting times of crystallization determined by DSC which are listed in Table I. We analyzed the time dependence of the integrated intensity in the induction period in the same way as in the case of PEN. In the first stage, the integrated intensity hardly increases as shown in the insets of Figs. 5, 6, and 7. In the second stage, each integrated intensity increases exponentially, but it somewhat depends on the annealing temperature in these cases. In the third stage, the increasing rate of each integrated intensity strongly depends on the annealing temperature. The annealing temperature dependence is very similar to the case of PEN crystallization. When crystallization starts, the integrated intensity also begins to increase more rapidly because of the growth of crystalline oriented domains as in the case of PEN. The steeper increasing intensities are consistent with the starting times of crystallization obtained by DSC measurements. It is therefore natural to consider that the increase of DPLS intensities after crystallization is caused by the growth of crystalline aggregates.

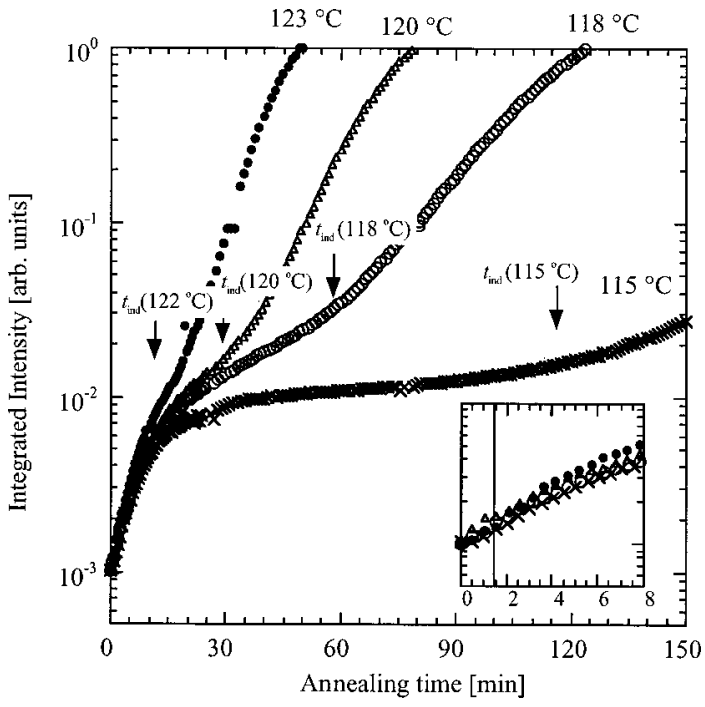

FIG. 6. Annealing time dependence of the integrated intensity of DPLS for sPS as a function of annealing temperature: $123(\mathbf{O}), 120$ $(\triangle), 118(\bigcirc)$, and $115^{\circ} \mathrm{C}(\times)$. Arrows indicate the times at which crystallization begins. The inset is the enlargement until the initial 8 min.

\section{DISCUSSION}

As described in the previous section, we have separated the induction period into three stages which are defined as the time domains corresponding to the initial step where the integrated intensity of DPLS hardly changes, the subsequent step where the integrated intensity grows exponentially with time, and the last step where the intensity levels off. In this section, we analyze the temperature dependences of the integrated intensities in these stages using Arrhenius equations in order to clarify the structure, formation processes during

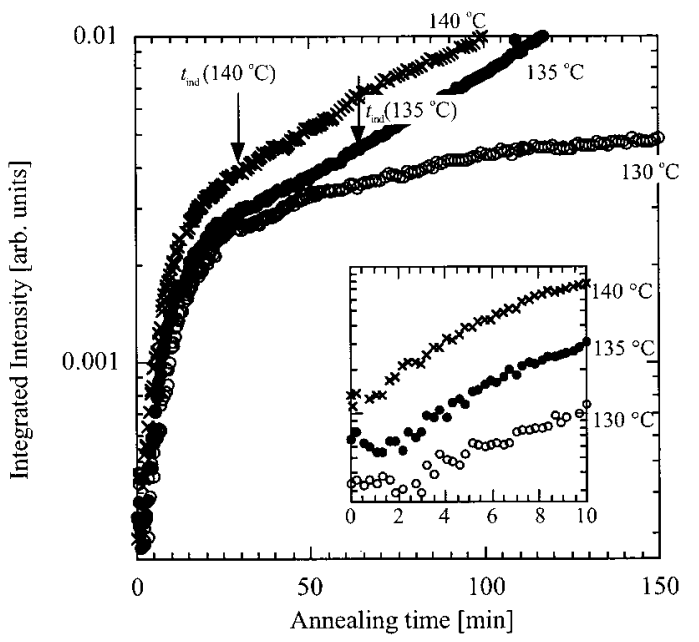

FIG. 7. Annealing time dependence of the integrated intensity of DPLS for iPS as a function of annealing temperature: $140(\times), 135$ (O), and $130{ }^{\circ} \mathrm{C}(\mathrm{O})$. Arrows indicate the times at which crystallization begins. The inset is the enlargement until the initial $10 \mathrm{~min}$. 

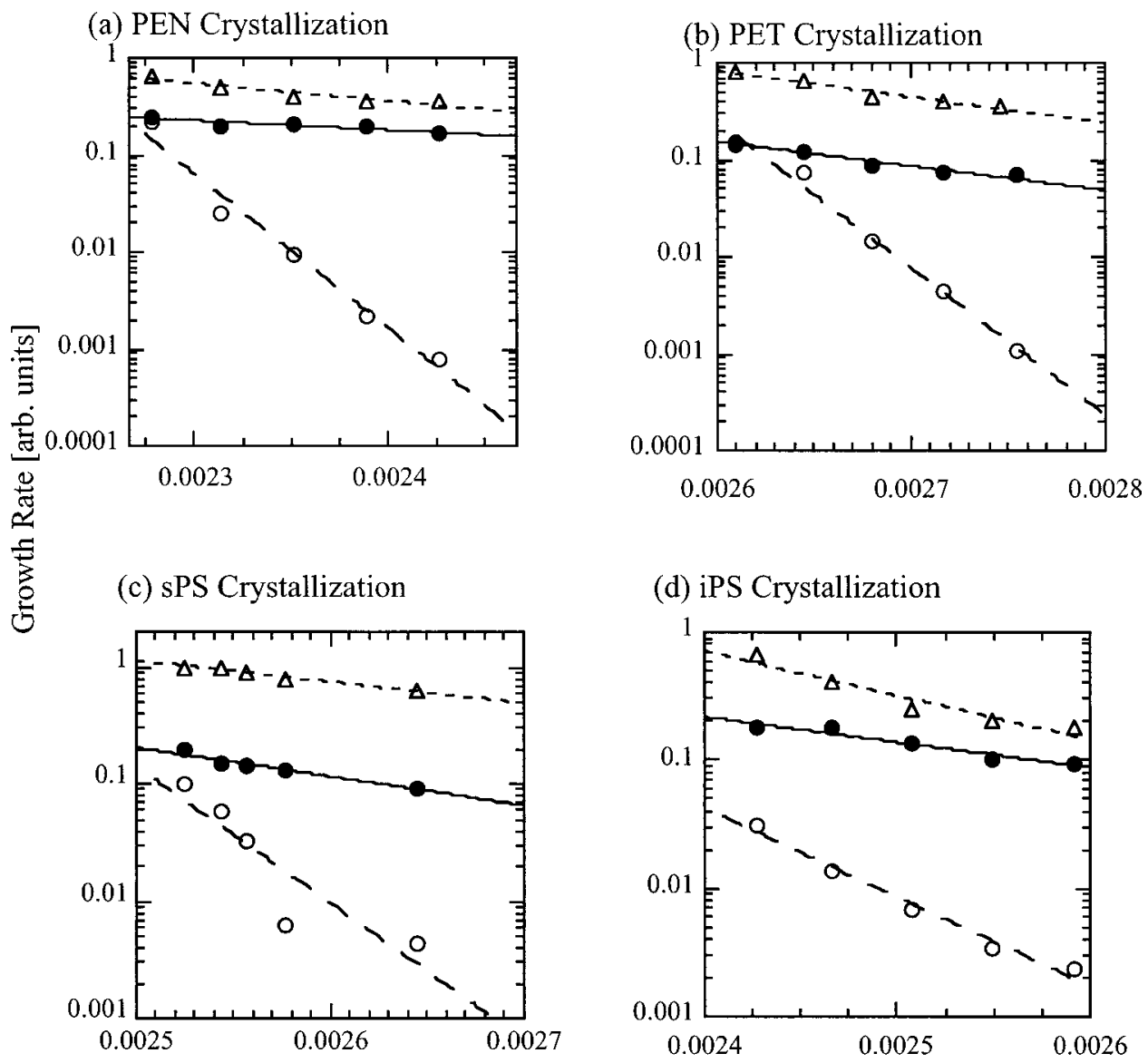

$T^{1}\left[\mathrm{~K}^{-1}\right]$

FIG. 8. Annealing temperature dependence of the growth rate for each stage for PEN (a), PET (b), sPS (c), and iPS (d). ( $\triangle$ ): the growth rate in the first stage, $(\mathbf{O})$ : in the second stage, and $(\bigcirc)$ : in the third stage.

the induction period. The physical meanings of these stages will be discussed later.

\section{Estimation of activation energies}

Here it is assumed that the rate $R_{1}$ corresponding to the first stage is proportional to a reciprocal of the length of the first stage, $t_{1}^{-1}$, i.e., $R_{1} \propto t_{1}^{-1}$ because in this stage orientational fluctuations hardly occur. Then, the activation energy, $\Delta E_{1}$, can be calculated by

$$
R_{1}=R_{10} \exp \left(-\Delta E_{1} / k_{\mathrm{B} T}\right)
$$

where $k_{\mathrm{B}}$ is the Boltzmann's factor and $R_{10}$ is a coefficient. Furthermore, it is assumed that both the rates $R_{2}$ and $R_{3}$ corresponding to the second and third stages are equal to the increasing rates of the integrated intensities: $R_{\chi}$ $=\partial I_{+}(t, T) / \partial t$. Then, the activation energies, $\Delta E_{\chi}$, for these stages are given also by Arrhenius-type plot

$$
R_{\chi}=R_{\chi 0} \exp \left(-\frac{\Delta E_{\chi}}{k_{\mathrm{B}} T}\right)
$$

where $R_{\chi}$ is the growth rate of the integrated intensity for the second $(x=2)$ or third $(x=3)$ stage of the induction period. In order to show the practical method how to estimate the growth rates for these stages, an example for PEN when annealed at $150{ }^{\circ} \mathrm{C}$ is given also in Fig. 3. The annealing temperature dependences of the growth rates for these three stages are shown for all the samples in Fig. 8, and the resulting activation energies are listed in Table II. Using these results we will discuss the three stages of structural formation process in the induction period below.

\section{First stage}

In the first stage of the induction period, the integrated intensity of DPLS hardly increases with time, suggesting that the systematic orientational ordering of polymer segments does not occur here. As was clarified by the time-resolved FTIR measurements of crystalline iPS, sPS, and PEN [1114], the conformational changes from random to helical conformations start to occur in the very early stage of the induction period before the beginning of chain segment orientation. Here, it should be noted that the partial segments having helical conformations correspond to rigid segments 
TABLE II. Apparent activation energies for three stages of the induction period of crystallization for PEN, PET, sPS, and iPS when annealed just above $T_{g}$.

\begin{tabular}{ccccc}
\hline \hline \multicolumn{5}{c}{ Activation Energy (kJ/mol) } \\
Stage polymer & PEN & PET & sPS & \multirow{2}{*}{ iPS } \\
\hline 1st stage & 39.0 & 40.4 & 34.9 & 37.1 \\
2nd stage & 24.8 & 34.9 & 46.0 & 50.0 \\
3rd stage & 406 & 296 & 222 & 177 \\
\hline \hline
\end{tabular}

being almost equal to crystalline conformations and generally the helical structure includes a planar zigzag conformation $(2 / 1$ helix).

From the results of the time-resolved FTIR measurements, we have concluded that the conformational changes begin in the first stage of the induction period of crystallization and the lengths of the rigid segments or the persistence lengths of the polymer chains increase in this stage $[11,12]$. However, the orientational fluctuations hardly occur in this time region as seen from the fact that the integrated intensity hardly increases. This means that in the first stage, the average length of the rigid segments does not attain a critical value for the isotropic-to-nematic transition predicted by Shimada, Doi, and Okano [7-10]. Furthermore, the activation energies for the chain segments to assume helical structure are in the range of 35 to $40 \mathrm{~kJ} / \mathrm{mol}$ or 8 to $10 \mathrm{kcal} / \mathrm{mol}$ for all the polymers as seen from Table II. These values correspond to three to four times as large as the potential barrier of a single $\mathrm{C}-\mathrm{C}$ bond rotation, meaning probably that three to four $\mathrm{C}-\mathrm{C}$ bonds need to rotate simultaneously in order to form the helical structure.

\section{Second stage}

As described previously, we assigned the second stage to the early stage of SD where the integrated intensity grows exponentially with annealing time, and hence, it clearly corresponds to the main process of segmental orientation. The activation energies for this stage, which are listed also in Table II, are in the range of 25 to $50 \mathrm{~kJ} / \mathrm{mol}$ or 6 to 12 $\mathrm{kcal} / \mathrm{mol}$. Such parallel orientation is considered the rotation around the normal to the segment axis, which couples with density fluctuations [7-10]. The apparent values of activation energy during the second stage for sPS and iPS are higher than those for PET and PEN. This may be because the formers have large side groups of benzene rings while the latters have no such side groups, i.e., because the orientation ordering of the rigid segments is correlated with their axial ratios; the larger the axial ratio, the faster the rate of orientation.

\section{Third stage}

In the third stage, the integrated DPLS intensity increases very slowly especially when annealed at lower temperatures, and the annealing temperature dependence of the integrated intensity is very strong for every sample. In the case of PEN when annealed at $155^{\circ} \mathrm{C}$, we notice that the third stage corresponds to the late stage of SD for the growth of density fluctuations detected by SAXS [13], which was confirmed with Furukawa's scaling theory $[18,19]$. However, it is unclear how the third stage of the DPLS change is related to the late stage of SD. In order to understand this relationship, let us discuss the activation energies for this stage.

As seen from Table II, the apparent activation energies for the third stage depend on the polymer species; those of crystalline polystyrenes (sPS and iPS) are about $200 \mathrm{~kJ} / \mathrm{mol}$ while those of PET and PEN are about 300 and $400 \mathrm{~kJ} / \mathrm{mol}$, respectively. Nevertheless, both of these values are by one order of magnitude larger than those for the other two stages. The reason for this may be understood in the framework of the theory by Binder and Stauffer [19] which we applied for the analysis of the SAXS data of PEN. Thus, the late stage of $\mathrm{SD}$ is dominated by the growth of dense or oriented domains (clusters) due to the cooperative process of diffusion and reaction, and the growth of such clusters may involve motions crossing very large potential barriers. For further details, the mechanism of the diffusion and reaction of clusters is visualized as follows. The diffusion occurs as a result of evaporation of atomic groups from the surface of a cluster and their subsequent condensation on the different site of the surface of the same cluster, resulting in the center of mass of the cluster shifts. Such diffusion of neighboring clusters causes their collision and fusion into larger clusters to reduce the surface energies.

In this connection we are reminded of the so-called "cooperatively rearranging regions (CRR)" of fragile liquids in the field of the glass transition since the apparent activation energies for the $\alpha$ process of fragile glass-forming materials are about $500 \mathrm{~kJ} / \mathrm{mol}$ near the glass transition temperature [20], which are of the same order as those for the third stage or the late stage of SD. Thus, we may assume that the evaporating atomic groups correspond to the atomic groups of the CRR. Then, what are their sizes? Here, we estimate the sizes of CRR from the apparent activation energies $E_{\text {app }}$ of the $\alpha$ process in terms of a conformer model proposed by Matsuoka and Quan [21], which is based on the Adam-Gibbs theory [22]. According to this model, the number of conformers $z$ in CRR is given by

$$
\begin{gathered}
E_{\text {app }}=\Delta \mu z, \\
\Delta \mu=\Delta \mu^{*} T^{*} /\left(T^{*}-T_{0}\right) .
\end{gathered}
$$

Here, $\Delta \mu$ is an activation energy for rearrangement of a conformer, and $T^{*}$ and $T_{0}$ are the highest- and lowesttemperature limits at which the conformer rearranges independently and cooperatively with all conformers in the system, respectively. $\Delta \mu^{*}$ and $T_{0}$ are related to the parameters $B$ and $T_{\mathrm{VF}}$ in the Vogel-Fulcher equation describing the relaxation time $\tau$ of the $\alpha$ process as a function of temperature

$$
\ln \left(\tau / \tau_{\infty}\right)=B /\left(T-T_{\mathrm{VF}}\right),
$$

where $\tau_{\infty}$ is a constant, $B=\Delta \mu * / k_{\mathrm{B}}$, and $T_{\mathrm{VF}}=T_{0}, k_{\mathrm{B}}$ being the Boltzmann constant and $T_{\mathrm{VF}}$ being called the VogelFulcher temperature. The parameters $B$ and $T_{\mathrm{VF}}$ can be experimentally evaluated by fitting the Vogel-Fulcher equation 
to the temperature dependence of the relaxation time of the $\alpha$ process. Values of $B$ and $T_{\mathrm{VF}}$ for PET [23] and atactic polystyrene (aPS) were found in the literature [21]: $B=745 \mathrm{~K}$ and $T_{\mathrm{VF}}=304 \mathrm{~K}$ for PET, and $B=1610 \mathrm{~K}$ and $T_{\mathrm{VF}}=323 \mathrm{~K}$ for aPS, respectively. The highest-temperature limit $T^{*}$ is assumed to be $773 \mathrm{~K}$ for both PET and aPS [21]. Using these values and the observed activation energies of the growth rate in the third stage listed in Table II, the numbers of conformers $z$ in CRR were evaluated. In this case, the parameters $B$ and $T_{\mathrm{VF}}$ of aPS were employed for those of iPS because the latter parameters were not found in literature. The resulting numbers of conformers are 7.7 and 29 for iPS and PET, respectively, in the temperature range of the present measurement. Here, it should be noted that a conformer is a part of a monomer in the polymer. The numbers of conformers in a monomer for PS and PET were estimated by Matsuoka and Quan [21] to be 2 and 5, which mean that the average numbers of monomers in CRR for iPS and PET are 3.8 and 5.8, respectively.

As described above, the activation energies or the sizes of CRR in the third stage for PEN and PET are larger than those for sPS and iPS. This may be because the interactions among polymer segments of PEN or PET are stronger than those of
sPS or iPS; in other words, the interactions among the naphthalene or benzene rings within the main chain are stronger than those among the side benzene groups.

In conclusion, we may conclude that in the third stage of the induction period the structure formation is described as diffusion and reaction processes of oriented domains, which is in harmony with the time evolution of the density fluctuations.

\section{CONCLUSION}

We have examined the structure formation processes during the induction period when crystallized from the glassy state within temperature ranges between $T_{g}$ and $T_{g}+50{ }^{\circ} \mathrm{C}$ from a viewpoint of orientation fluctuations by performing the time-resolved depolarized light-scattering (DPLS) measurements for PEN, PET, sPS, and iPS. These results revealed that the induction period can be separated into three stages; the first stage where the helical conformations are formed, the second stage corresponding to the early stage of SD where the parallel orientation of the helical (rigid) segments starts to occur, and the third stage corresponding to the late stage of SD, where the orientated domains grow by their diffusion and reaction.
[1] M. Imai, K. Mori, K. Mizukami, K. Kaji, and T. Kanaya, Polymer 33, 4451 (1992).

[2] M. Imai, K. Mori, K. Mizukami, K. Kaji, and T. Kanaya, Polymer 33, 4457 (1992).

[3] M. Imai and K. Kaji, Ordering Process in the Induction Period of Polymer Crystallization (Kotaibutsuri, Japan, 1997) Vol. 32, No. 6, pp. 449-458.

[4] M. Imai, K. Kaji, T. Kanaya, and Y. Sakai, Phys. Rev. B 52, 12696 (1995).

[5] M. Imai, K. Kaji, T. Kanaya, and Y. Sakai, Physica B 213, 718 (1995).

[6] M. Imai, K. Kaji, and T. Kanaya, Phys. Rev. Lett. 71, 4162 (1992).

[7] M. Doi and S. F. Edwards, The Theory of Polymer Dynamics (Oxford University, New York, 1986), Chap. 10, which may be referred to in addition to the following original literature [8-10].

[8] T. Shimada, M. Doi, and K. Okano, J. Chem. Phys. 88, 2815 (1988).

[9] M. Doi, T. Shimada, and K. Okano, J. Chem. Phys. 88, 4070 (1988).
[10] T. Shimada, M. Doi, and K. Okano, J. Chem. Phys. 88, 7181 (1988).

[11] G. Matsuba, K. Kaji, K. Nishida, T. Kanaya, and M. Imai, Macromolecules 32, 8932 (1999).

[12] G. Matsuba, K. Kaji, K. Nishida, T. Kanaya, and M. Imai, Polym. J. (Tokyo) 31, 722 (1999).

[13] G. Matsuba, T. Kanaya, M. Saito, K. Kaji, and K. Nishida, Phys. Rev. E 62, R1497 (2000).

[14] G. Matsuba, T. Kanaya, K. Kaji, and K. Nishida, Abstract of IUPAC Macro 2000 (Wiley-VCH, Berlin, 2000).

[15] J. Koberstain, T. P. Russel, and R. S. Stein, J. Polym. Sci., Polym. Phys. Ed. 17, 1719 (1979).

[16] J. W. Cahn and J. E. Hilliard, J. Chem. Phys. 28, 258 (1958).

[17] J. W. Cahn, J. Chem. Phys. 42, 93 (1965).

[18] H. Furukawa, Physica A 123, 497 (1984).

[19] K. Binder and D. Stauffer, Phys. Rev. Lett. 33, 1006 (1974).

[20] M. D. Ediger, C. A. Angell, and S. R. Nagel, J. Phys. Chem. 100, 13200 (1996).

[21] S. Matsuoka and X. Quan, Macromolecules 24, 2770 (1991).

[22] G. Adam and J. H. Gibbs, J. Chem. Phys. 43, 139 (1965).

[23] A. Hoffmann, Ph.D thesis, Mainz University, 1993. 